\title{
On the strength of a system of partial differential equations
}

\author{
Bernard F. Schutz \\ Department of Applied Mathematics and Astronomy, University College, Cardiff, Wales \\ (Received 4 November 1974) \\ It is shown that Einstein's concept of the "strength" of a system of differential equations is directly \\ related to the number of dynamical degrees of freedom the equations permit. It may become a useful \\ tool for investigating the structure of the system.
}

In a recent paper, Mariwalla ${ }^{1}$ discussed Einstein' $s^{2}$ concept of the "strength" of a system of partial differential equations. Although both suggested that the strength was related to the amount of arbitrariness in the solutions to the system, neither Mariwalla nor Einstein made that relationship satisfactorily quantitative, and both were surprised to find that the Maxwell and Einstein equations had the same strength, twice that of the scalar wave equation. The purpose of this paper is to show that the strength is related in a well-defined manner to the number of arbitrary functions of $d-1$ variables (where $d$ is the dimension of the manifold) necessary to determine a solution locally. For hyperbolic systems this is the amount of Cauchy data; it defines the amount of dynamical freedom in the system. The strengths of the scalar, Maxwell, and Einstein systems are then readily understood in terms of the number of polarization states available to the massless particles associated with them. When extended to examine arbitrariness in fewer than $d-1$ variables, the method may become a useful tool in examining the structure of the system of equations.

Suppose we have a system of equations for $n$ unknowns $U_{A}$ on a $d$-dimensional manifold. If all the equations can be placed in the normal form

$$
\partial^{k} U_{A} / \partial x^{k}=f_{A}\left(x, y_{\alpha}, \partial^{l+m} U_{B} / \partial^{l} x \partial^{m} y_{B}\right)
$$

[where $\left\{x, y_{\alpha}(\alpha=1, \ldots, d-1)\right\}$ are the $d$ coordinates, $k$ is fixed, and $l+m \leqslant k, l<k]$ in some coordinate system, then a local analytic solution is always uniquely determined by giving analytic functions for $U_{A}$ and their first $k-1$ derivatives with respect to $x$ in the hypersurface $x=$ const (Cauchy-Kowalewsky theorem). That is, the sytem allows $k n$ free functions of $k-1$ variables. However, it may not always be possible to choose coordinates $\left\{x, y_{\alpha}\right\}$ such that all the equations of the system take the form (1). In the Maxwell or Einstein equations, for example, some equations represent constraints, of the form

$$
0=g_{A}\left(x, v_{\alpha}, \partial^{l+m} U_{B} / \partial^{l} x \partial^{m} y_{B}\right)
$$

Associated with the existence of these contraints are gauge functions, whose values are arbitrary everywhere. These gauge functions are physically unmeasurable, but must be eliminated before the Cauchy-Kowalewsky theorem can be brought to bear on the problem. The existence of the constraints, moreover, means that not all the initial data is freely specifiable. We shall show that Einstein's concept of the "strength" of the system provides a direct, if somewhat heuristic, method of discovering just how much real freedom there is in a complicated system of partial differential equations.

Consider the Taylor expansion of an analytic function of $d$ variables about a point. The total number of terms of $n$th order in the expansion is ${ }^{1}$

$$
\left[\begin{array}{l}
d \\
n
\end{array}\right] \equiv \frac{(n+d-1) !}{n !(d-1) !}=\left(\begin{array}{c}
n+d-1 \\
n
\end{array}\right) .
$$

If the function is completely unconstrained, then all of these coefficients in the Taylor series may be given arbitrarily. If for any reason the function can be given arbitrarily on a $(d-1)$-dimensional hypersurface, but its behavior in the remaining dimension is determined, then only $\left[\begin{array}{c}d-1 \\ n\end{array}\right]$ coefficients of order $n$ are arbitrary. The converse is not necessarily true (the $\left[\begin{array}{c}d^{-1} \\ n\end{array}\right]$ free coefficients need not form a $(d-1)$-dimensional Taylor expansion), but in the context in which we shall use it we can suppose it will generally be true. The fraction of free coefficients in such a function is

$$
\left[\begin{array}{c}
d-1 \\
n
\end{array}\right] /\left[\begin{array}{l}
d \\
n
\end{array}\right]=\frac{d-1}{n+d-1},
$$

which goes to zero for large $n$.

In order to determine the amount of freedom in a system of partial differential equations, Einstein ${ }^{2}$ suggested one should expand all the dependent variables in Taylor series and determine the number of relations among the various coefficients of order $n$ that are implied by the differential equations of the system. By subtraction there remains a number $Z_{n}$ of free coefficients of order $n$. Einstein ${ }^{2}$ and Mariwalla ${ }^{1}$ have computed this number for several physically interesting fields. After removing all the gauge freedom in the fields, they found that the ratio $Z_{n} /\left[\begin{array}{l}d \\ n\end{array}\right]$ always went to zero as $1 / n$ for large $n$, and they interpreted this to mean that there were no completely free functions of $d$ variables left in the theories. They then defined the coefficient of $1 / n$ to be the "strength," but did not interpret it satisfactorily. It is clear, however, from the discussion of the previous paragraph, that the limit for large $n$ of $Z_{n} /\left[\begin{array}{c}i-1 \\ n\end{array}\right]$, which differs from the Einstein strength by a factor of $(d-1)$, is in fact the number of free functions of $d-1$ variables in the theory. We can 
formulate this precisely as follows. It is clear from Eq. (3) that $\left[\begin{array}{l}d \\ n\end{array}\right]$ is a polynomial in $n$ of order $d-1$. Since $Z_{n}$ is always a sum of such terms, ${ }^{1,2}$ it is also a polynomial in $n$ of maximum order $d-1$. Therefore, it has a unique representation of the form

$$
Z_{n}=\sum_{k=1}^{k} N_{k}\left[\begin{array}{l}
k \\
n
\end{array}\right]
$$

Then, at least heuristically, the number of free functions of $k$ variables in the solution is $N_{k}$. (In the Appendix we show that all $N_{k}$ are integers, as they must be for this approach to make sense.)

As a concrete example, let us consider the Maxwell field in four dimensions. When the equations are formulated in terms of a vector potential and the gauge freedom is removed from $Z_{n}$ explicitly, one obtains ${ }^{1}$

$$
Z_{n}^{(1)}=4\left[\begin{array}{l}
4 \\
n
\end{array}\right]-\left[\begin{array}{c}
4 \\
n+1
\end{array}\right]-4\left[\begin{array}{c}
4 \\
n-2
\end{array}\right]+\left[\begin{array}{c}
4 \\
n-3
\end{array}\right] \text {. }
$$

If we were not to subtract the gauge freedom, the second term would not be present. If the vector potential is not introduced at all, one obtains ${ }^{1,2}$

$$
Z_{n}^{(2)}=6\left[\begin{array}{l}
4 \\
n
\end{array}\right]-8\left[\begin{array}{c}
4 \\
n-1
\end{array}\right]+2\left[\begin{array}{c}
4 \\
n-2
\end{array}\right] \text {. }
$$

Writing these in the form of Eq. (4) gives

$$
\begin{aligned}
& Z_{n}{ }^{(1)}=4\left[\begin{array}{l}
3 \\
n
\end{array}\right]-2\left[\begin{array}{l}
2 \\
n
\end{array}\right]-2\left[\begin{array}{l}
1 \\
n
\end{array}\right], \\
& Z_{n}{ }^{(2)}=4\left[\begin{array}{l}
3 \\
n
\end{array}\right]+2\left[\begin{array}{l}
2 \\
n
\end{array}\right] .
\end{aligned}
$$

Neither $Z_{n}$ contains any free functions of four variables, and both have four free functions of three variables. These correspond to the two dynamical degrees of freedom in electromagnetism: On a Cauchy hypersurface one can specify two variables and their time derivatives freely. On the other hand, the two versions of Maxwell's equations appear to differ at the two- and one-dimensional level. In fact they do not: Because $Z_{n}{ }^{(1)}$ refers to a potential version of the $Z_{n}{ }^{(2)}$ equations, a term of order $n$ in the latter is of order $n+1$ in the former. To "lower" the order of the former, we rewrite $Z_{n}^{(1)}$ in terms of $m \equiv n-1$ and find

$$
Z_{m}^{(1)}=4\left[\begin{array}{c}
3 \\
m
\end{array}\right]+2\left[\begin{array}{c}
2 \\
m
\end{array}\right] \text {. }
$$

So in this sense both versions are equivalent. The interpretation of this two-dimensional term is not at all clear, but in view of its independence of the use of a potential, it appears to contain some real information about Maxwell's equations. We may conjecture that it describes the freedom to set a boundary condition on the elliptical constraint equation in the initial hypersurface, but it should bear a more thorough investigation. There is another tantalizing suggestion in the Einstein equations of general relativity, for which $Z_{n}{ }^{1,2}$ takes the form

$$
Z_{n}{ }^{\mathrm{GR}}=4\left[\begin{array}{l}
3 \\
n
\end{array}\right]-2\left[\begin{array}{l}
2 \\
n
\end{array}\right]-8\left[\begin{array}{l}
1 \\
n
\end{array}\right]
$$

This is derived using the metric tensor as the fundamental variable; but the metric may be considered to be a second-order potential for the Riemann tensor, which is physically measureable. Shifting the order by two $(m \equiv n-2)$, we get

$$
Z_{m}^{\mathrm{GR}}=4\left[\begin{array}{c}
3 \\
m
\end{array}\right]+6\left[\begin{array}{c}
2 \\
m
\end{array}\right] \text {. }
$$

The coefficient of $\left[\begin{array}{l}1 \\ m\end{array}\right]$ is zero, and the other coefficients are positive, just as for electromagnetism.

Whatever the significance of the lower coefficients may be, it is clearly not surprising that for the Einstein and Maxwell equations, as well as for the Weyl and Dirac equations, ${ }^{1}$ one finds $N_{3}=4$, while for the scalar wave equation $N_{3}=2$. All are field theories for spinning particles: for zero spin particles there is only one dynamical degree of freedom, while for massless particles with spin there are two possible helicities and hence two degrees of freedom.

\section{APPENDIX}

The expressions given by Einstein ${ }^{2}$ and Mariwalla ${ }^{1}$ are always of the form

$$
Z_{n}=\sum_{\substack{\text { integer } \\
m}} K_{m}\left[\begin{array}{c}
d \\
n-m
\end{array}\right] \text {, }
$$

where $d$ is the dimension of the manifold and the $K_{m}$ are always integers. This is in fact the general form for $Z_{n}$, because each term results from $K_{m}$ equations (always in $d$ dimensions) containing $m$ derivatives of the field variables. We wish to reexpress this in the form of Eq. (4). By using the definition of $\left[\begin{array}{l}d \\ n\end{array}\right]$ it is easy to derive the basic relation

$$
\left[\begin{array}{c}
d \\
n+1
\end{array}\right]=\left[\begin{array}{l}
d \\
n
\end{array}\right]+\left[\begin{array}{l}
d-1 \\
n+1
\end{array}\right], \quad d \geqslant 2
$$

By iteration of this we find

$$
\left[\begin{array}{c}
d \\
n+1
\end{array}\right]=\left[\begin{array}{l}
d \\
n
\end{array}\right]+\left[\begin{array}{c}
d-1 \\
n
\end{array}\right]+\left[\begin{array}{c}
d-2 \\
n
\end{array}\right]+\cdots+\left[\begin{array}{l}
1 \\
n
\end{array}\right] .
$$

Equation (A1) can be rewritten as

$$
\left[\begin{array}{c}
d \\
n-1
\end{array}\right]=\left[\begin{array}{l}
d \\
n
\end{array}\right]-\left[\begin{array}{c}
d-1 \\
n
\end{array}\right]
$$

from which follows by iteration

$$
\left[\begin{array}{c}
d \\
n-m
\end{array}\right]=\sum_{p=0}^{m}(-1)^{p}\left(\begin{array}{c}
m \\
p
\end{array}\right)\left[\begin{array}{c}
d-p \\
n
\end{array}\right] \text {. }
$$

Since all the coefficients are integers, the coefficients $N_{k}$ in Eq. (4) are also integers.

${ }^{1}$ K. H. Mariwalla, J. Math. Phys. 15, 468 (1974). Note the error in Eq. (23), where $\left(\frac{d}{2}\right)$ should everywhere be replaced by $\left(\begin{array}{c}d+1 \\ 2\end{array}\right)$.

${ }^{2}$ A. Einstein, Meaning of Relativity (Methuen, London, 1956), 6th ed., Appendix II; also (Princeton U. P. , Princeton, N.J., 1955), 5th ed. 